\title{
Clinical and diagnostic importance of dyslipidemia in children and adolescents during the coronavirus disease 2019 pandemic
}

\author{
Eun Young Kim, MD, PhD \\ Department of Pediatrics, Chosun University College of Medicine, Gwangju, Korea
}

\begin{abstract}
Key message
- Because childhood lipid concentrations continue into adulthood, early evaluation and treatment are needed, but dyslipidemia awareness is low.

- For the prevention and treatment of dyslipidemia in childhood and adolescence, a major risk factor for cardiovascular disease in adulthood, lifestyle modifications, appropriate exercise, and drug treatment are required.

- A large-scale study of the prevalence and therapeutic effects of dyslipidemia in children and adolescents in Korea is needed.
\end{abstract}

Dyslipidemia in children and adolescents is a known major risk factor for cardiovascular disease in adulthood along with obesity.1) In particular, since childhood lipid concentrations continue into adulthood, the early evaluation and treatment of dyslipidemia are required during childhood and adolescence. ${ }^{2)}$ Therefore, it is necessary to detect children and adolescents at risk of cardiovascular disease in adulthood by screening for dyslipidemia.

The Korean Society of Pediatric Endocrinology guidelines, based on National Heart, Lung, and Blood Institute recommendations, state that all children and adolescents aged 9-11 and 17-21 years of age require dyslipidemia screening, even if asymptomatic or lacking a family history. Testing for dyslipidemia is not recommended for children under 2 years of age. Routine screening tests are not performed for children aged 2-8 years and those 12-16 years of age. However, in this age group, if there is a family history of high-risk cardiovascular disease and dyslipidemia or risk factors such as hypertension, diabetes, and chronic kidney disease, targeted screening can be performed. ${ }^{3,4)}$ In addition, non-high-density lipoprotein cholesterol, which does not require fasting as a screening test and can predict cardiovascular disease in adulthood, is recommended. ${ }^{5)}$

Although the prevalence of dyslipidemia and obesity are increasing in children and adolescents in Korea, ${ }^{6}$ awareness of dyslipidemia as well as education about its prevention and treatment are insufficient.
This article reviews studies showing that dyslipidemia in children and adolescents is associated with the cardiovascular system as well as vitamin D levels, respiratory disease, and mental health. ${ }^{7}$ Although the results of published studies are inconsistent, they are expected to be helpful in the recognition of serious health problems caused by dyslipidemia in children and adolescents.

Studies have reported that social distancing and activity restrictions implemented during the coronavirus disease 2019 (COVID-19) pandemic have exacerbated metabolic syndrome, including obesity and dyslipidemia, as a result of decreased physical activity and increased unhealthy food intake in adults. ${ }^{8)}$ Similarly, a recently published study in Korea reported that the prevalence of obesity, dyslipidemia, and vitamin D deficiency among children and adolescents are increasing due to school closures and physical activity restrictions during the COVID-19 pandemic, ${ }^{9)}$ increasing awareness of the importance of health care for children and adolescents.

The increased prevalence of dyslipidemia is considered due to a westernized diet and reduced physical activity levels. Therefore, it is essential to change lifestyles by increasing the consumption of healthy foods, promoting breakfast, and decreasing screen time and regular activities for the treatment of dyslipidemia. ${ }^{10)}$ Although studies have examined the relationship between dyslipidemia and vitamin $\mathrm{D}$, it is unclear whether vitamin $\mathrm{D}$ administration helps improve dyslipidemia. The primary treatment for obesity or dyslipidemia is lifestyle modification; however, it may be necessary to exercise indoors and take supplemental vitamin D to prevent cardiovascular disease and support bone health due to social distancing and the restriction of outdoor activities during the COVID-19 pandemic. ${ }^{9)}$ Drug treatment for dyslipidemia can be performed in children aged 10 years or older whose conditions do not improve despite lifestyle modifications and diet control for 6-12 months. Statins have been approved by the U.S. Food and Drug Administration for the first-line treatment of dyslipidemia in children aged 10 years and older. In Korea, almost $0.41 \%$ of children and adolescents

Corresponding author: Eun Young Kim, MD, PhD. Department of Pediatrics, Chosun University Hostpital, Chosun University College of Medicine, 365 Pilmun-daero, Dong-gu, Gwangju 61453, Korea 
with dyslipidemia require drug treatment. ${ }^{11)}$

To enable more effective evaluations or treatments of dyslipidemia in children and adolescents, it is necessary to select risk groups through a life-cycle health checkup program and utilize a system that can continuously provide individual preventive education and customized treatment in connection with medical institutions. There is also a need to develop better guidelines for the management of dyslipidemia through management system feedback. A large-scale integrated study is also needed because of the rarity of epidemiological statistics or drug treatment studies of dyslipidemia in children and adolescents in Korea.

See the article "A new perspective on cholesterol in pediatric health: association of vitamin D metabolism, respiratory diseases, and mental health problems" via https://doi.org/10.3345/cep. 2020.00934 .

\section{Footnote}

Conflicts of interest: No potential conflict of interest relevant to this article was reported.

\section{References}

1. Berenson GS, Srinivasan SR, Bao W, Newman WP 3rd, Tracy RE, Wattigney WA. Association between multiple cardiovascular risk factors and atherosclerosis in children and young adults. The Bogalusa Heart Study. NEngl J Med 1998;338:1650-6.

2. Lauer RM, Clarke WR. Use of cholesterol measurements in childhood for the prediction of adult hypercholesterolemia. The Muscatine Study.
JAMA 1990;264:3034-8.

3. Lim JS, Kim EY, Kim JH, Yoo JH, Yi KH, Chae HW, et al. 2017 Clinical practice guidelines for dyslipidemia of Korean children and adolescents. Ann Pediatr Endocrinol Metab 2020;25:199-207.

4. Expert Panel on Integrated Guidelines for Cardiovascular Health and Risk Reduction in Children and Adolescents; National Heart, Lung, and Blood Institute. Expert panel on integrated guidelines for cardiovascular health and risk reduction in children and adolescents: summary report. Pediatrics 2011;128(Suppl 5):S213-56.

5. Srinivasan SR, Frontini MG, Xu J, Berenson GS. Utility of childhood non-high-density lipoprotein cholesterol levels in predicting adult dyslipidemia and other cardiovascular risks: the Bogalusa Heart Study. Pediatrics 2006;118:201-6.

6. Song K, Kim H, Chae HY. Prevalence and treatment of pediatric dyslipidemia. J Korean Med Assoc 2021:64;410-5.

7. Hong J. A new perspective on cholesterol in pediatric health: association of vitamin D metabolism, respiratory diseases, and mental health problems. Clin Exp Pediatr 2022;66;65-72.

8. Sohn M, Koo BK, Yoon HI, Song KH, Kim ES, Kim HB, et al. Impact of COVID-19 and associated preventive measures on cardiometabolic risk factors in South Korea. J Obes Metab Syndr 2021;30:248-60.

9. Kang HM, Jeong DC, Suh BK, Ahn MB. The impact of the coronavirus disease-2019 pandemic on childhood obesity and vitamin D status. J Korean Med Sci 2021;36:e21.

10. Elkins C, Fruh S, Jones L, Bydalek K. Clinical practice recommendations for pediatric dyslipidemia. J Pediatr Health Care 2019;33:494-504.

11. Yang S, Hwang JS, Park HK, Lee HS, Kim HS, Kim EY, et al. Serum lipid concentrations, prevalence of dyslipidemia, and percentage eligible for pharmacological treatment of Korean children and adolescents; data from the Korea National Health and Nutrition Examination Survey IV (2007-2009). PLoS One 2012;7:e49253.

How to cite this article: Kim E. Clinical and diagnostic importance of dyslipidemia in children and adolescents during the coronavirus disease 2019 pandemic. Clin Exp Pediatr 2022; 65:129-30. https://doi.org/10.3345/cep.2021.01851 